\title{
Large-Collection-Angle BF STEM Imaging of Compound Semiconductors
}

\author{
T. Aoki ${ }^{1}$, M.R. McCartney ${ }^{2}$, and David J. Smith ${ }^{2}$ \\ ${ }^{1}$ LeRoy Eyring Center for Solid State Science, Arizona State University, Tempe, AZ 85287-1704, USA \\ ${ }^{2}$ Department of Physics, Arizona State University, Tempe, AZ 85287-1504, USA
}

The aberration-corrected scanning transmission electron microscopy (AC-STEM) is a powerful and versatile instrument, which can be equipped with a multitude of detectors including high-angle annulardark-field (HAADF), medium-angle annular-dark-field (MAADF), bright-field (BF), annular-brightfield $(\mathrm{ABF})$, secondary electron (SE), and back-scattered (BSE), as well as energy-dispersive $\mathrm{x}$-ray spectrometer (EDS) and electron-energy-loss spectrometer (EELS). Because HAADF STEM is an incoherent imaging mode with high atomic-number sensitivity ("Z-contrast"), and because it can be easily combined with EELS, it is most often utilized. However, modern AC STEMs can acquire multiple images from different detectors simultaneously, and these other imaging modes can also provide valuable materials information. Because each mode can be considered as complementary, the acquisition of several images allows better understanding and interpretation of materials microstructure. Phase contrast dominates in $\mathrm{BF}$ imaging when a small collection angle is used, as implied by the reciprocity theorem. However, when the collection angle is increased to be as large as the convergence semi-angle, phase contrast is suppressed and incoherent contributions dominate image formation [1].

In recent studies of compound semiconductor heterostructures [2,3], we have discovered the usefulness of large-collection-angle bright-field (LABF) STEM imaging, especially when used in combination with HAADF imaging. Here we describe and compare images of $\mathrm{GaSb}$, $\mathrm{ZnTe}$ and GaAs, which were acquired with all three materials in [110]-type orientations, using a JEOL ARM-200F operated at $200 \mathrm{kV}$. The LABF and HAADF images were taken with a convergence semi-angle of $21 \mathrm{mrad}$, the BF collection angle was $21 \mathrm{mrad}$, and the HAADF collection angle was 90mrad 220mrad. Figures 1(a) - (d) show $\mathrm{LABF}$ and HAADF images of GaSb (where $\mathrm{Z}_{\mathrm{Ga}}=31$ and $\mathrm{Z}_{\mathrm{Sb}}=51$ ), as well as corresponding intensity profiles. Figures $1(\mathrm{e})$ - (h) show images and line profiles for $\mathrm{ZnTe}$ (where $\mathrm{Z}_{\mathrm{Zn}}=30$ and $\mathrm{Z}_{\mathrm{Te}}=$ 52), and Figures 1(i) - (l) show GaAs (where $Z_{\mathrm{Ga}}=31$ and $Z_{\mathrm{As}}=33$ ). With the convergence semi-angle equal to the BF collection angle, phase contrast was suppressed, no contrast reversals were observed, and LABF and HAADF images were both in-focus at the same time. In LABF, the positions of the atomic columns appear with dark contrast and show improved visibility, where atomic columns occupied by heavier atoms exhibit darker contrast than columns occupied by lighter atoms. When the $\mathrm{Z}$ difference is large (i.e., GaSb and ZnTe), the atomic columns of lighter element become blurred out in the HAADF image, yet they were clearly observed in the corresponding LABF, as seen by comparing Figs. 1 (a) and (c), and Figs. 1 (e) and (g). It is significant that even small Z-differences can be detected in LABF images, as shown by the case of GaAs and its intensity profile in Figs. 1(i) and (j). Overall, it is concluded that LABF STEM is another powerful AC-STEM imaging mode, with improved Zsensitivity, that is highly suited for studying complicated heterostructures at the atomic scale [4].

References:

1. J. Liu and J. M. Cowley, Ultramicroscopy 52 (1993) 335-346.

2. D. J. Smith, et al., Microscopy 62 (2013) S65-S73.

3. D.J. Smith, et al., J. Phys. Conf. Ser. 471 (2013) 012005.

4. We acknowledge the use of facilities in the John M. Cowley Center for High Resolution Electron Microscopy at Arizona State University. 

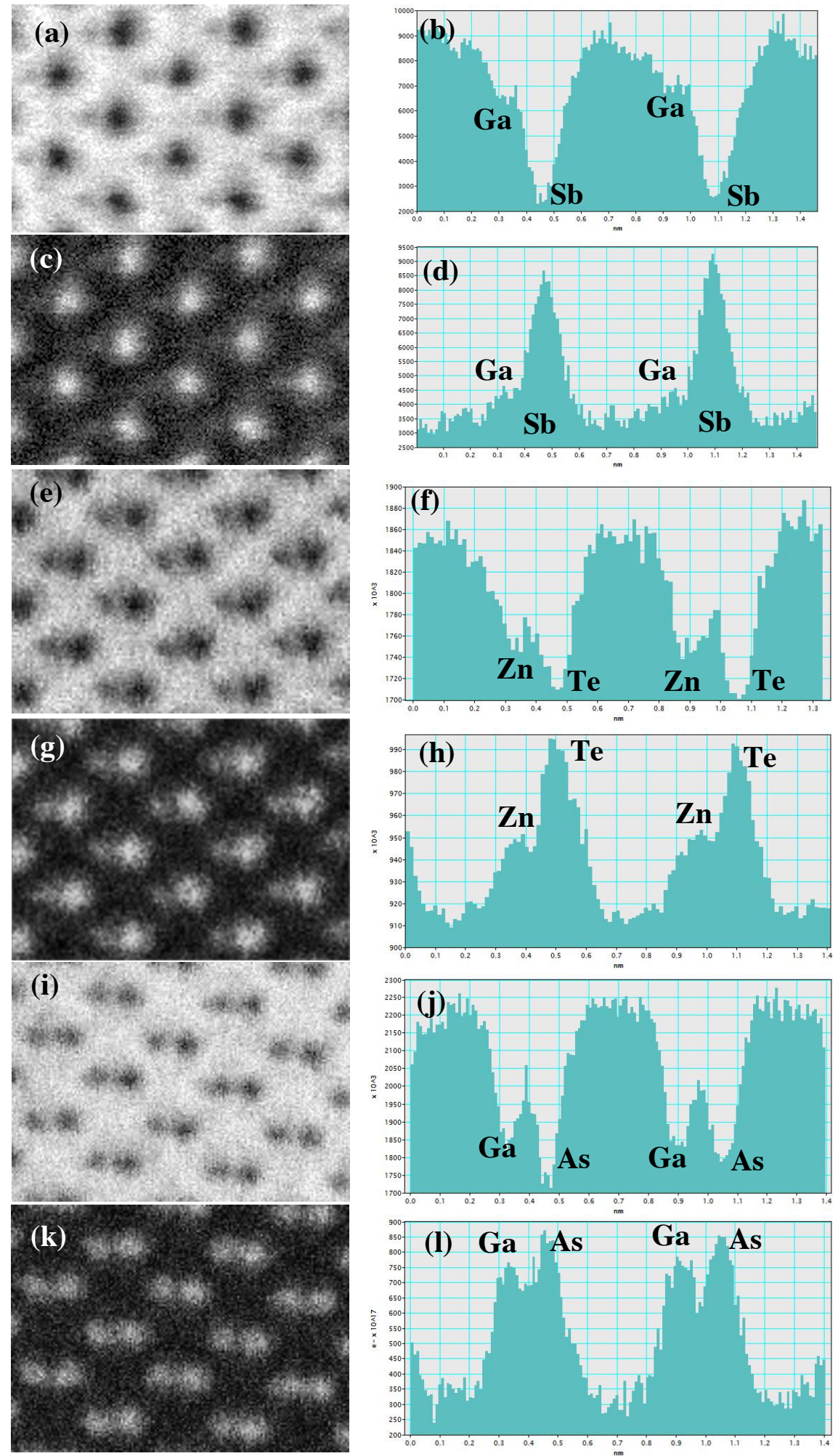

Fig. 1. (a) GaSb LABF image; (b) intensity profile of GaSb LABF image; (c) GaSb HAADF; (d) intensity profile of GaSb HAADF image; (e) ZnTe LABF image; (f) intensity profile of ZnTe LABF image; (g) ZnTe HAADF image; (h) intensity profile of ZnTe HAADF image; (i) GaAs LABF image; (j) intensity profile of GaAs LABF image; (k) GaAs HAADF image; (l) intensity profile of GaAs HAADF image. 